\title{
Optimization of Eva Green real-time mPCR for differentiating C. jejuni/coli directly from feces
}

\author{
Pavlova $\mathrm{M}^{1}$, Velev $\mathrm{V}^{2}$, Dobreva $\mathrm{E}^{1}$, Asseva $\mathrm{G}^{1}$, Ivanov $\mathrm{I}^{1}$, Petrov $\mathrm{P}^{1}$, Mangarov $\mathrm{A}^{2}$, Tomova $\mathrm{I}^{2}$, \\ Kantardjiev $\mathrm{T}^{1}$
}

National Centre of Infectious and Parasitic Diseases, Sofia, Bulgaria. velev_md@abv.bg

\begin{abstract}
OBJECTIVE: To develop and optimize a rapid molecular method for diagnosing campylobacteriosis directly from a clinical fecal sample and at the same time for determining the most common causing agents $-C$. jejuni/coli. MATERIALS AND METHODS: 38 clinical fecal samples from hospitalized patients with diarrheal syndrome were tested using a rapid immunochromatographic test. All positive samples were tested for confirmation by culturing in a microaerophilic atmosphere. The Eva Green real-time mPCR reaction of a direct fecal sample was conducted using the "IQ5TM Real-Time PCR System" apparatus.

RESULTS: Out of 38 clinical fecal samples which were ICT positive, 18 strains were isolated by culture, namely, 17 of $C$. jejuni and 1 of $C$. coli. The Eva Green real-time MPCR reaction also reported 18 positive samples for Campylobacter, out of which 17 were of $C$. jejuni and only one of C.coli.

CONCLUSION: We developed and optimized the Eva Green real-time mPCR for the detection and species differentiation of $C$. jejuni/coli directly from a clinical fecal sample. The molecular analysis we described has a $100 \%$ sensitivity and specificity when comparing the results obtained by it to those of the culture method, which is currently the "gold standard" in the diagnosis of campylobacteriosis (Tab. 2, Fig. 1, Ref. 6). Text in PDF www.elis.sk. KEY WORDS: Campylobacter, diarrhea, mPCR.
\end{abstract}

\section{Introduction}

Enteropathogenic bacteria are the cause of acute infectious diarrhea, which is a global health problem (3-6). In our country, routine tests for Campylobacter spp. are not yet performed on diarrheal patients, and if done, it is by using traditional culture methods, which are slow, laborious and capricious. Very rarely the microbiological laboratories in Bulgaria perform species characteristics of the most common strains of C. jejuni and C. coli, thus making epidemiological studies on campylobacteriosis difficult $(1,4)$.

\section{Materials and methods}

For the period of August 2016 - October 2016, 38 clinical fecal samples of hospitalized patients aged between 0 and 7 years and suffering from diarrheal syndrome at the Hospital for Infectious Diseases, Sofia were tested. The clinical samples were tested using a rapid immunochromatographic test (ICT) (CerTest Biotec) for the Campylobacter antigen. All positive samples were tested for confirmation using culturing. The cultures were

${ }^{1}$ National Centre of Infectious and Parasitic Diseases, Sofia, Bulgaria, and ${ }^{2}$ Department of Infectious Diseases, Parasitology and Tropical Medicine, Medical University of Sofia, Bulgaria

Address for correspondence: $\mathrm{V}$. Velev, MD, Medical University of Sofia, Bulv. "G. Sofiiski" 1, Sofia, Bulgaria. grown in a microaerophilic atmosphere generated by a gas pack in an anaerobic jar (Oxoid USA) at $42-43{ }^{\circ} \mathrm{C}$ for $24-72$ hours. The isolates were biochemically differentiated by a positive reaction of sodium hippurate hydrolysis and/or positive reaction of indoxyl acetate hydrolysis for C. jejuni and positive reaction of indoxyl acetate hydrolysis, and negative reaction of sodium hippurate hydrolysis for C. coli.

DNA extraction. Bacterial DNA from the clinical fecal samples was extracted using a ready-for-use kit QUIampDNABloodMiniKit (QUIAGEN, Germany) in accordance with the manufacturer's instructions (Eva Green real-time multiplex PCR). The approved PCR method for simultaneous detection and differentiation of C. jejuni/coli directly from a fecal sample is based on three pairs of primers, which until now were not included together in a common PCR reaction: primers - AB F2/R2, amplifying a specific region of the cj041 gene for $C$. jejuni, ceuE $\mathbf{F} / \mathbf{R}$, amplifying a specific region of the ceuE gene for $C$. Coli, and primers pair camp F2/R2, amplifying a specific region of the genus gene for Campylobacter spp. The primers used and their sequences are provided in Table 1 . Two reference strains are also included, namely 33559ATCC for C. coli and 335560 of $C$. jejuni, used as positive controls and $\mathrm{ddH}_{2} 0$ used as a negative control (Tab. 1).

The Eva Green real-time mPCR analysis is optimized to be conducted in a final reaction volume of $25 \mu 1$ at the specified primers and conditions $1 \mathrm{x}$ Taq DNA Polymerase buffer; $2.5 \mathrm{x}$ DMSO; 0.2 mMdNTP; 0.03/ $\mu$ l HS Taq DNA Polymerase (Appli- 
Tab. 1. Sequence of the primers used and the size of their amplicons.

\begin{tabular}{|c|c|c|}
\hline Gene & Sequence & Amplicon \\
\hline \multirow[t]{2}{*}{$16 \mathrm{~S}$ rDNA } & CACGTGCTACAATGGCATAT & $108 \mathrm{bp}$ \\
\hline & GGC+TTCATGCTCTCGAGTT & \\
\hline ceuE- F & CAAGTACTGCAATAAAAACTAGCACTACG & $72 \mathrm{bp}$ \\
\hline ceuE- $R$ & AGCTATCACCCTCATCACTCATACTAATAG & \\
\hline cj041-F & GATACCTTAAGTGCAGCCTGTGA & $74 \mathrm{bp}$ \\
\hline cj041-R & ACGCCTAAACCTATAGCTCCTTC & \\
\hline
\end{tabular}

chem GmbH, Germany); 0,005 $\mu$ Mcamp - F2/R2; $0.55 \mu$ MceuE $-\mathrm{F} / \mathrm{R} ; 0.4 \mu \mathrm{MAB}-\mathrm{F} / \mathrm{R}$.

The Eva Green real-time $\mathrm{mPCR}$ reaction takes place in an "IQ5TM Real-Time PCR System" under the following conditions: the first step is denaturation for $10 \mathrm{~min}$. at $95{ }^{\circ} \mathrm{C}$, followed by 29 cycles, each consisting of $15 \mathrm{sec}$. of denaturation at $90^{\circ} \mathrm{C}, 20 \mathrm{sec}$. of $58^{\circ} \mathrm{C}$ hybridization and $25 \mathrm{sec}$. of $60^{\circ} \mathrm{C}$ elongation; while the latter final step of elongation lasted $5 \mathrm{~min}$. at $72^{\circ} \mathrm{C}$. The resulting amplicons measure $108 \mathrm{bp}, 72 \mathrm{bp}$ and $74 \mathrm{bp}$ in size, while these DNA fragments correspond to the camp genes for Campylobacter spp., CeuE for C. coli and cj0414 for C. jejuni.

Determining the analytical sensitivity and specificity of the Eva Green real-time mPCR method

For determining the analytical specificity, a group of commonly isolated gastroenteritis-causing bacteria was selected, particularly Y.enterocolitica, E.coli stx1d, E. coli O104, Sh. flexneri, S. Typhi, Cl. difficile and S. aureus toxC. The strains ATCC 33560 and C-14.2 were used as a positive control for C. jejuni and $C$. coli, respectively. A 10x DNA of the cultures of the respective strains was used.

For determining the analytical sensitivity, a series of dilutions of a tenfold bacterial suspension of C. jejuni and C. coli in saline was used. Volumes of $10 \mu 1$ of each dilution were used to artificially inoculate feces which were negative for campylobacter. The QUIampDNABloodMiniKit was used for DNA extraction.

\section{Results and discussion}

All fecal samples were tested for $C$. jejuni/coli using three separate methods: ICT, culture method and using the Eva Green real-time mPCR. The results obtained are provided in Table 2.

A rapid immunochromatographic test (ICT) for the Campylobacter antigen has been performed on clinical samples of patients with diarrheal syndrome aged between 0 and 7 years and hospitalized at the Hospital for Infectious Diseases, Sofia, and all positive

Tab. 2. Positive results of the three laboratory methods used to diagnose campylobacteriosis.

\begin{tabular}{lcc}
\hline ICT $(+)$ & Culture method $(+)$ & mPCR $(+)$ \\
\hline 37 & 18 & 18 \\
\cline { 2 - 3 } & C. jejuni -17 & C. jejuni -17 \\
\cline { 2 - 3 } & C. coli -1 & C. coli -1 \\
\hline
\end{tabular}

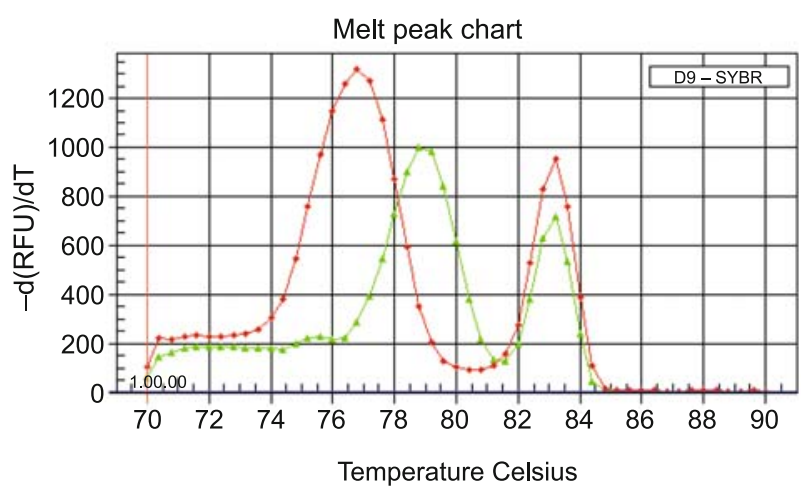

Fig. 1. Analysis of the peaks of amplification after a Real-time mPCR at $\mathrm{T}_{\mathrm{a}}=58^{\circ} \mathrm{C}$. C. jejuni $\left(\mathrm{AB}=78.8^{\circ} \mathrm{C}\right), \mathrm{C}$. coli $\left(\operatorname{ceuE}=76.8^{\circ} \mathrm{C}\right)$.

samples have been verified with a culture and molecular test at the Centre for Infectious and Parasitic Diseases, Sofia.

Out of 38 fecal samples which were ICT positive, 18 strains were isolated in a culture; 17 of $C$. jejuni and 1 of $C$. coli. This analysis provides a rapid and more reliable detection of bacterial cells compared to the conventional culture methods using biochemical differentiation. The amplification curves of the products can be monitored in real time, and the melting temperatures typical of the target genes are respectively: $78.80{ }^{\circ} \mathrm{C}$ for $C$. jejuni, $76.80{ }^{\circ} \mathrm{C}$ for C. coli and $82.80^{\circ} \mathrm{C}$ for Campylobacter spp. at the optimal melting temperature of the primers $\left(\mathrm{T}_{\mathrm{a}}\right)$ of $58^{\circ} \mathrm{C}$, based on which we optimized the protocol (Fig. 1).

Out of 38 clinical samples tested for $C$. jejuni/coli, the PCR analysis reported 18 positive samples for Campylobacter while in 17 of them the PCR products had the typical melting temperatures for $C$. jejuni and only one for C. coli. All other samples were negative for Campylobacter spp.

Determining the analytical sensitivity of the Eva Green real-time mPCR method.

In each PCR reaction for analytical sensitivity we used $7.5 \mu \mathrm{l}$ of concentrated DNA extracted from artificially inoculated feces with serial dilutions of $10 \mu \mathrm{l}$ of a tenfold bacterial suspension. The analytical sensitivity of the test is approximately $103 \mathrm{CFU}$ per 150 $\mu 1$ of liquid stool. This analytical sensitivity is significantly higher than any published to date (2).

The molecular analysis we described has a $100 \%$ sensitivity and specificity when comparing the results obtained by it to those of the culture method, which is currently the "gold standard" in the diagnosis of campylobacteriosis. In this study, the PCR method is inferior to ICT only in terms of speed.

\section{Conclusion}

The fecal samples of patients with acute enterocolitis may contain a variety of pathogenic bacteria. For the purposes of early initiation of etiological treatment, the rapid detection of the bacterial agent causing the particular disease and providing a timely 


\section{2-704}

diagnosis are important. We developed the Eva Green real-time mPCR analysis as a rapid, sensitive and specific method for the diagnosis of campylobacteriosis directly from a clinical fecal sample and at the same time determined the two most frequently isolated species - C. jejuni and C. coli for the needs of molecular epidemiology. This method will be very useful in emergency situations such as in controlling epidemic situations, pediatric infectology, as well as in immunosuppressed patients.

\section{Reference}

1. Boyanova L, Gergova G, Spassova $\mathbf{Z}$ et al. Campylobacter infection in 682 bulgarian patients with acute enterocolitis, inflammatory bowel disease, and other chronic intestinal diseases. Diagn Microbiol Infect Dis 200449 (1): 71-74.
2. Center of Disease Control. 2008. Campylobacter.http://www.cdc.gov./ nezved/disease_listing/Campylobacter_gi.html.

3. Daskalov H, Maramski A. Prevalence and factors affecting the presence of Campylobacter spp. in broiler carcasses in Bulgaria. Turk J Vet Anim Sci 2012; 36 (5): 539-545.

4. Stoicheva M, Velcheva R, Murdjeva $M$ et al. Intestinal infections in children under 5 years, Medicart 3; 10: 23-26.

5. Sahin O, Kassem II, Shen Z et al. Campylobacter in Poultry: Ecology and Potential Interventions. Avian Dis 2015; 59 (2): 185-200.

6. World Health Organization, 2008, Campylobacter Fact Sheet \#255, http://www.who.int./mediacentre/factsheets/fs255/en/

Received May 4, 2017. Accepted May 22, 2017. 